\title{
Variational integrators for orbital problems using frequency estimation
}

DOI:

$10.1007 /$ s10444-018-9603-y

\section{Document Version}

Accepted author manuscript

Link to publication record in Manchester Research Explorer

\section{Citation for published version (APA):}

Kosmas, O., \& Leyendecker, S. (2019). Variational integrators for orbital problems using frequency estimation. Advances in Computational Mathematics, 45(1), 1-21. https://doi.org/10.1007/s10444-018-9603-y

\section{Published in:}

Advances in Computational Mathematics

\section{Citing this paper}

Please note that where the full-text provided on Manchester Research Explorer is the Author Accepted Manuscript or Proof version this may differ from the final Published version. If citing, it is advised that you check and use the publisher's definitive version.

\section{General rights}

Copyright and moral rights for the publications made accessible in the Research Explorer are retained by the authors and/or other copyright owners and it is a condition of accessing publications that users recognise and abide by the legal requirements associated with these rights.

\section{Takedown policy}

If you believe that this document breaches copyright please refer to the University of Manchester's Takedown Procedures [http://man.ac.uk/04Y6Bo] or contact uml.scholarlycommunications@manchester.ac.uk providing relevant details, so we can investigate your claim.

\section{OPEN ACCESS}




\title{
Variational integrators for orbital problems using frequency estimation
}

\author{
Odysseas Kosmas • Sigrid Leyendecker
}

Received: date / Accepted: date

\begin{abstract}
In this work, we present a new derivation of higher order variational integration methods that exploit the phase lag properties for numerical integrations of systems with oscillatory solutions. More specifically, for the derivation of these integrators, the action integral along any curve segment is defined using a discrete Lagrangian that depends on the endpoints of the segment and on a number of intermediate points of interpolation. High order integrators are then obtained by writing down the discrete Lagrangian at any time interval as a weighted sum of the Lagrangians corresponding to a set of the chosen intermediate points. The respective positions and velocities are interpolated using trigonometric functions. The methods derived this way depend on a frequency, which in general needs to be accurately estimated. The new methods, which improve the phase lag characteristics by re-estimating the frequency at every time step, are presented and tested on the general $N$-body problem as numerical examples. variational integrators; discrete variational mechanics; highly oscillatory problems; phase lag analysis; general $N$-body problem.
\end{abstract}

\section{Introduction}

One of the most difficult problems in numerical solution of ordinary differential equations is the development of integrators for highly oscillatory systems, see [1]. Standard numerical schemes may require a huge number of time steps to track the oscillations, and even with small size steps they can alter the

Odysseas Kosmas

Modelling and Simulation Centre, MACE, University of Manchester, Sackville Street, Manchester, UK

E-mail: odysseas.kosmas@manchester.ac.uk

Sigrid Leyendecker

Chair of Applied Dynamics, University of Erlangen-Nuremberg Erlangen, 91058, Germany 
dynamics, unless the method is chosen adequately. In such cases, it is useful to adopt geometric integrators, that is, numerical schemes which preserve some of the geometric features of the dynamical system. These integrators can run in simulations for long time with less spurious effects (for instance, bad energy behavior for conservative systems) than the traditional ones, see [2-9].

So far, special techniques have been developed to improve the numerical integration of highly oscillatory problems, e.g. [1], and to derive methods as well as error bounds for families of quadrature methods that use finite difference approximations for the required derivatives. Alternatively, using the phase-lag property of [10], trigonometric fitting and phase-fitting techniques lead to methods based on variable coefficients which depend on the characteristic frequency of the problem, see [11]. The latter technique is known as exponential (or trigonometric) fitting and has been formulated long ago, see $[10,12,13]$. Exponentially fitting algorithms are considered as natural extensions of the classical polynomial fitting due to their characteristic property to approach the classical ones as the involved dominant frequencies tend to zero. The important problem of convergence of exponentially fitted methods, especially of the known as multi-step ones, has been investigated within Lyche's theory in [13] (for a comprehensive discussion the reader is referred to $[10,14]$ ).

By exploiting the main benefits of the phase fitting and variational integrators, in our previous work [16] we constructed adaptive time step variational integrators. We used a Lagrangian problem similar to that employed for testing ordinary differential equations, e.g. the harmonic oscillator with a given frequency $\omega$. Subsequently, the phase fitted variational integrators, which solve exactly the test system, were applied to a general Lagrangian problem of single particle motion (the planar two body problem) by determining the frequency $\omega$ at every step of the integration.

In the present work, by combining the above techniques (using interpolation procedures) we generalize these integrators and construct high order methods using phase fitted techniques with a constant time step, for the numerical solution of the general $N$-body problem. Contrary to our previous method [16], in the present paper we introduce different frequency expressions for each individual body. Numerical examples show that the estimation of each body's frequency is needed at every step of the integration for the methods using trigonometric interpolating functions, see e.g. [17].

The paper is organized as follows. After giving a brief overview of variational integrators and the phase lag technique, in Sections 2 and 3, in Section 4 interpolation processes are employed for the derivation of variational integrators of high order. They are, then, tested through the solution given for the simple harmonic oscillator in Section 5. Due to the fact that, such numerical results rely on the definition of a parameter $u$, Section 6 is devoted for the realization of this parameter by using phase lag analysis. The resulting methods are tested on the orbital motion of one body and also on the general $N$-body problem in Sections 7 and 8. Finally, the conclusion extracted are summarized in Section 9. 


\section{Review of variational integrators}

The least action principle of the continuous Lagrange dynamics can be used as a guiding principle to derive discrete integrators. Following the steps of the derivation of the Euler-Lagrange equations in the continuous time, one can derive the discrete time Euler-Lagrange equations, see [4]. For this purpose, one need consider a positive time step $h \in \mathbb{R}$ and configurations $q_{k}, q_{k+1}$ approximating the continuous trajectory $q:\left[t_{0}, t_{N}\right] \rightarrow Q$ at time nodes $t_{k}$ and $t_{k+1}=t_{k}+h$ as $q_{k} \approx q\left(t_{k}\right), q_{k+1} \approx q\left(t_{k+1}\right)$, where $(q, \dot{q}) \in T Q$ live in the tangent bundle of the configuration manifold $Q$, which here is considered to be smooth and finite dimensional.

The discrete Lagrangian $L_{d}: Q \times Q \rightarrow \mathbb{R}$ approximates the action integral along the curve segment between $q_{k}$ and $q_{k+1}$, i.e.

$$
L_{d}\left(q_{k}, q_{k+1}\right) \approx \int_{t_{k}}^{t_{k+1}} L(q, \dot{q}) d t
$$

where e.g. $L_{d}\left(q_{k}, q_{k+1}\right)=h L\left(\frac{q_{k}+q_{k+1}}{2}, \frac{q_{k+1}-q_{k}}{h}\right)$. Hence, in the discrete setting the correspondence to the state space $T Q$ is $Q \times Q$. An intuitive motivation for this is that two points close to each other correspond approximately to the same information as one point and a velocity vector. The next step is to compute the discrete action along the discrete trajectory $\left\{q_{k}\right\}_{k=0}^{N}$ by

$$
S_{d}=\sum_{k=0}^{N-1} L_{d}\left(q_{k}, q_{k+1}\right) .
$$

Following the case of the continuous dynamics, we compute variations of this action sum with the boundary points $q_{0}$ and $q_{N}$ held fixed. The discrete Hamilton's principle states that the motion of the discrete mechanical system extremizes the action sum, i.e. $\delta S_{d}=0$. By differentiating and rearranging the terms and having in mind that both $q_{0}$ and $q_{N}$ are fixed, the discrete Euler-Lagrange (DEL) equations are obtained, see [4]

$$
D_{2} L_{d}\left(q_{k-1}, q_{k}\right)+D_{1} L_{d}\left(q_{k}, q_{k+1}\right)=0, \quad k=1, \ldots, N-1
$$

where the notation $D_{i} L_{d}$ indicates the slot derivative with respect to the $i$ argument of $L_{d}$.

The definition of the discrete conjugate momentum at time steps $k$ and $k+1$ reads

$$
p_{k}=-D_{1} L_{d}\left(q_{k}, q_{k+1}\right), \quad p_{k+1}=D_{2} L_{d}\left(q_{k}, q_{k+1}\right), \quad k=0, \ldots, N-1
$$

The latter definitions can be used for the initialization of the two step integrator (3), see Sections 5 and 7.2 . 


\section{Phase fitting technique}

The features of the phase fitting technique emerge out of its application to first order ordinary differential equations, e.g. to the test problem

$$
\frac{d y(t)}{d t}=i \omega y(t), y(0)=1
$$

which has exact solution of oscillatory type

$$
y(t)=e^{i \omega t}
$$

For the regularity, domain and range of the function $y(t)$ see [15]. The main advantages of the phase fitting can also be shown from its application to second order differential equations as that of a harmonic oscillator

$$
\frac{d^{2} y(t)}{d t^{2}}+\omega^{2} y(t)=0
$$

with exact solution

$$
y(t)=A e^{i \omega t}+B e^{-i \omega t} .
$$

For any of the solutions (6) and (8), the values at a time $t+h$ must be determined, which e.g. for the solution of $(6)$ reads

$$
y(t+h)=e^{i \omega h} y(t)
$$

assuming that all past values $y(t)$ are exactly known. Then, the numerical estimation of $y(t+h)$ of $(9)$, denoted by $\hat{y}(t+h)$, which is an approximate solution, may be written as

$$
\hat{y}(t+h)=\alpha(\omega h) e^{i \phi(\omega h)} y(t) .
$$

In the latter expression, $\alpha(\omega h)$ represents the amplification error and $\phi(\omega h)$ appears in the definition of the phase lag. Under these assumptions, the ratio of the estimated to the exact solution in (10) and (9) takes the form

$$
\frac{\hat{y}(t+h)}{y(t+h)}=\alpha(\omega h) e^{-i \ell(\omega h)} .
$$

In the latter equation $\ell(\omega h)=\omega h-\phi(\omega h)$ is called phase lag of the numerical approximation $\hat{y}(t+h)$. In the case when $\alpha(\omega h)=1$ and $\ell(\omega h)=0$, we say that the numerical approximation is exponentially fitted at the frequency $\omega$ and at the time step $h$, see also [16].

Hence, the goal of the phase fitting technique is to minimize the phase lag while simultaneously forcing $\alpha(\omega h)$ to tend, as closely as possible, to unity, see [10]. 


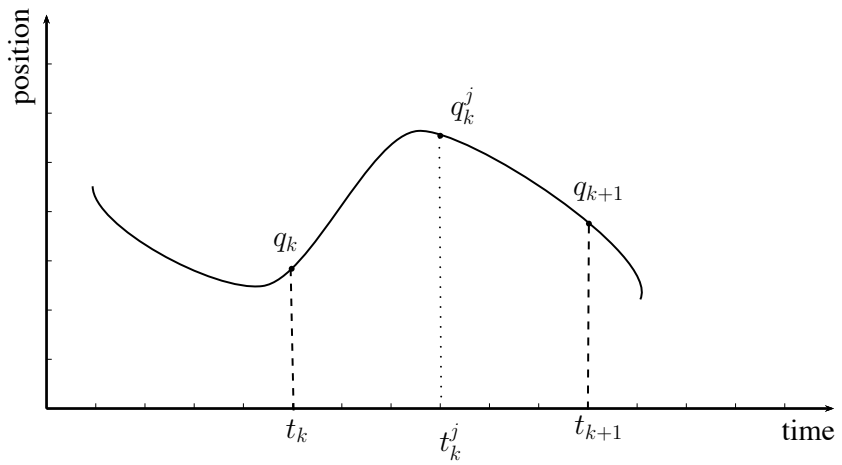

Fig. 1: Intermediate time nodes $t_{k}^{j} \in\left[t_{k}, t_{k+1}\right]$ and corresponding configurations $q_{k}$ and $q_{k+1}$.

\section{Discrete Lagrangian using interpolation techniques}

For the derivation of high order methods based on variational integrators for the solution of physical problems general interpolation techniques can be applied. We first consider problems with separable Lagrangians i.e. Lagrangians where kinetic energy $T$ and potential energy $U$ depend only on the generalized velocity $\dot{q}$ and the generalized position $q$, respectively (a case of velocity dependent potential is the Lorentz force field discussed in [23]).

As mentioned in Section 2, the action integral along the curve segment between $q_{k}$ and $q_{k+1}$ (see Figure 1) is expressed by using a discrete Lagrangian that depends on these configurations and on a number of intermediate configurations $q_{k}^{j}$ obtained via interpolation. For this reason, for $Q=\mathbb{R}^{d}, d \in \mathbb{N}$, we obtain expressions for configurations $q_{k}^{j}$ and velocities $\dot{q}_{k}^{j}$ (for $j=0, \ldots, S-1$, $S \in \mathbb{N}$ ) at time $t_{k}^{j} \in\left[t_{k}, t_{k+1}\right]$ by expressing $t_{k}^{j}=t_{k}+C_{k}^{j} h$ for $C_{k}^{j} \in[0,1]$ such that $C_{k}^{0}=0, C_{k}^{S-1}=1$ and applying the interpolation, see [16]

$$
\begin{aligned}
q_{k}^{j} & =g_{1}\left(t_{k}^{j}\right) q_{k}+g_{2}\left(t_{k}^{j}\right) q_{k+1} \\
\dot{q}_{k}^{j} & =\dot{g}_{1}\left(t_{k}^{j}\right) q_{k}+\dot{g}_{2}\left(t_{k}^{j}\right) q_{k+1} .
\end{aligned}
$$

The functions $g_{1}\left(t_{k}^{j}\right)$ and $g_{2}\left(t_{k}^{j}\right)$ are chosen for the intended type of the interpolation (see Sections 4.1 and 4.2). Furthermore the following conditions must hold

$$
\begin{aligned}
g_{1}\left(t_{k+1}\right) & =0, & g_{2}\left(t_{k}\right) & =0 \\
g_{1}\left(t_{k}\right) & =1, & g_{2}\left(t_{k+1}\right) & =1 .
\end{aligned}
$$

For any chosen number of intermediate points $S$, using weights $w^{j}$, we define the discrete Lagrangian by the weighted sum

$$
L_{d}\left(q_{k}, q_{k+1}\right)=h \sum_{j=0}^{S-1} w^{j} L\left(q\left(t_{k}^{j}\right), \dot{q}\left(t_{k}^{j}\right)\right),
$$


where, as can be easily proved, for maximal algebraic order the following conditions must hold

$$
\sum_{j=0}^{S-1} w^{j}\left(C_{k}^{j}\right)^{m}=\frac{1}{m+1}, \quad m=0,1, \ldots, S-1, \quad k=0,1, \ldots, N-1
$$

for $C_{k}^{j}=\frac{t_{k}^{j}-t_{k}}{h}$. We here refer to maximal algebraic order as the maximal number of coefficients of the Taylor expansion of the right hand side of equation (14) when compared to the discrete Lagrangian of the harmonic oscillator, see also [16]. Since this expression does not depend on the functions $g_{1}$ and $g_{2}$ of (12), i.e. on the type of interpolation, the expression for the coefficients $w^{j}$ must hold for any interpolation method employed.

\subsection{Linear and cubic spline interpolation}

We first consider the linear interpolation in the interval $\left[t_{k}, t_{k+1}\right]$ for a function $q_{k}^{j} \approx q\left(t_{k}^{j}\right)$, which is given by the formula

$$
q_{k}^{j}=A_{k}^{j} q_{k}+B_{k}^{j} q_{k+1}
$$

where

$$
A_{k}^{j}=\frac{t_{k+1}-t_{k}^{j}}{h}, \quad B_{k}^{j}=1-A_{k}^{j}=\frac{t_{k}^{j}-t_{k}}{h} .
$$

The above expressions are a special case of the general Lagrange interpolation formula, see [21].

In the case when variational integrators result by using linear interpolation, $g_{1}$ and $g_{2}$ in (12) are defined as

$$
g_{1}\left(t_{k}^{j}\right)=1-\frac{t_{k}^{j}-t_{k}}{h}, \quad g_{2}\left(t_{k}^{j}\right)=\frac{t_{k}^{j}-t_{k}}{h} .
$$

The later expressions fulfil the properties (13).

Since it is (piecewise) linear, $q_{k}^{j}$ of (16) has zero second derivative in the interior of each interval, and an undefined, or infinite, second derivative at the abscissas $t_{k}$. In contrast cubic spline interpolation yields a formula that is smooth in the first derivative and continuous in the second derivative, both within a time interval and at its boundaries.

If we further suppose that, in addition to the tabulated values of $t_{k}^{j}$, we also have tabulated values for the second derivatives, $\ddot{q}$, i.e. a set of numbers $\ddot{q}_{k}$, then, within each interval, we add to the right-hand side of (16) a cubic polynomial whose second derivative varies linearly from a value $\ddot{q}_{k}$ on the left to a value $\ddot{q}_{k+1}$ on the right. Under these circumstances, the desired continuous second derivative is obtained. If we further construct the cubic polynomial to have zero values at $t_{k}$ and $t_{k+1}$, adding them into (16) does not spoil the 
condition (13). It can be shown that $([21])$ there is only one way to arrange this construction, namely replacing (16) by

$$
q_{k}^{j}=A_{k}^{j} q_{k}+B_{k}^{j} q_{k+1}+D_{k}^{j} \ddot{q}_{k}+E_{k}^{j} \ddot{q}_{k+1}
$$

where $A_{k}^{j}$ and $B_{k}^{j}$ have been defined in (17) and

$$
D_{k}^{j}=\frac{1}{6}\left[\left(A_{k}^{j}\right)^{3}-A_{k}^{j}\right] h^{2}, \quad E_{k}^{j}=\frac{1}{6}\left[\left(B_{k}^{j}\right)^{3}-B_{k}^{j}\right] h^{2} .
$$

Notice here that, the dependence on the variable $t$ in (19) and (20) is entirely through the linear $t$-dependence of $A_{k}^{j}$ and $B_{k}^{j}$, and (through $A_{k}^{j}$ and $B_{k}^{j}$ ) the cubic $t$-dependence of $D_{k}^{j}$ and $E_{k}^{j}$, see [21].

Remark 1 For separable Lagrangians, the continuous Euler-Lagrange equations relate the acceleration and the configuration at each time. Thus, $\ddot{q}_{k}=$ $\ddot{q}_{k}\left(q_{k}\right)$ and $\ddot{q}_{k+1}=\ddot{q}_{k+1}\left(q_{k+1}\right)$ can be expressed in terms of the unknown configurations $q_{k}$ and $q_{k+1}$, respectively. Inserting them into (19) yields an expression for $q_{k}^{j}$ that depends only on the unknowns $q_{k}, q_{k+1}$.

Following the assumptions of Section 4, the configuration $q_{k}^{j}$ can be obtained using the cubic interpolation (19) while the parameters of the interpolation are given by the expressions (17) and (20). If we rewrite the expression of $q_{k}^{j}$ for the case of the numerical solution of a harmonic oscillator with frequency $\omega$ (see Section 5), the interpolating functions $g_{1}$ and $g_{2}$ take the form

$$
\begin{aligned}
& g_{1}\left(t_{k}^{j}\right)=1-\frac{t_{k}^{j}-t_{k}}{h}-\frac{1}{6}\left[\left(1-\frac{t_{k}^{j}-t_{k}}{h}\right)^{3}-\left(1-\frac{t_{k}^{j}-t_{k}}{h}\right)\right] h^{2} \omega^{2} \\
& g_{2}\left(t_{k}^{j}\right)=\frac{t_{k}^{j}-t_{k}}{h}-\frac{1}{6}\left[\left(\frac{t_{k}^{j}-t_{k}}{h}\right)^{3}-\frac{t_{k}^{j}-t_{k}}{h}\right] h^{2} \omega^{2} .
\end{aligned}
$$

As a numerical example for the integrators derived using linear and cubic spline interpolation, see Section 5 .

\subsection{Interpolation using trigonometric functions}

In the case of oscillatory problems, the use of an interpolation formula that depends on trigonometric functions can improve dramatically the behavior of the integrators, see for example [22].

For this case, interpolation using trigonometric functions can be obtained by assuming that $q_{k}^{j}$ are expressed as in (12) with $g_{1}, g_{2}$ being trigonometric functions having the properties (13).

A special case of these functions is given by

$$
g_{1}\left(t_{k}^{j}\right)=\frac{\sin \left(u-\frac{t_{k}^{j}-t_{k}}{h} u\right)}{\sin u}, \quad g_{2}\left(t_{k}^{j}\right)=\frac{\sin \left(\frac{t_{k}^{j}-t_{k}}{h} u\right)}{\sin u}
$$




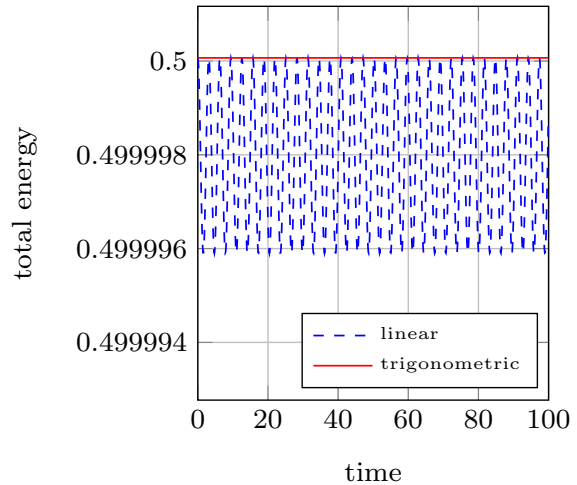

(a)

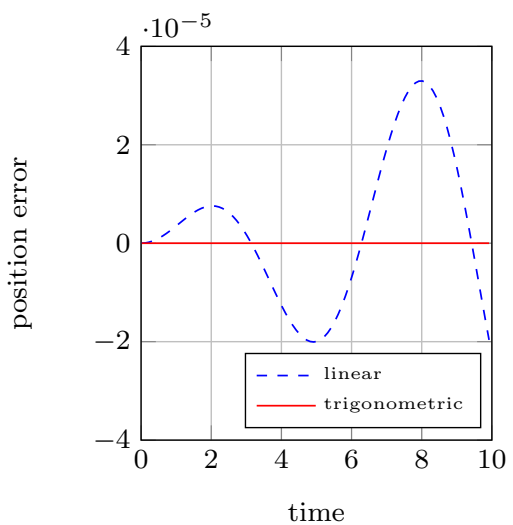

(b)

Fig. 2: Harmonic oscillator with frequency $\omega=1$, time step $h=0.01$ and $S=5$. (a): Evolution of the total energy for trigonometric interpolation derived with $u=\omega h$ versus linear interpolation. (b): Evolution of the position error for trigonometric interpolation derived with $u=\omega h$ and linear interpolation.

for $u=\in \mathbb{R} \backslash\{k \pi, k \in \mathbb{Z}\}$. Thus we have

$$
\begin{aligned}
& q_{k}^{j}=\frac{\sin \left(u-\frac{t_{k}^{j}-t_{k}}{h} u\right)}{\sin u} q_{k}+\frac{\sin \left(\frac{t_{k}^{j}-t_{k}}{h} u\right)}{\sin u} q_{k+1} \\
& \dot{q}_{k}^{j}=\frac{1}{h}\left[-\frac{u \cos \left(u-\frac{t_{k}^{j}-t_{k}}{h} u\right)}{\sin u} q_{k}-\frac{u \cos \left(\frac{t_{k}^{j}-t_{k}}{h} u\right)}{\sin u} q_{k+1}\right] .
\end{aligned}
$$

The derivation of the expressions (23) is described in [16,17].

It is now clear that the new interpolating functions depend on a parameter $u$. By changing this parameter different interpolation schemes can be obtained.

In the next section, we first present results for different numerical schemes obtained with arbitrary parameters $u$ for the solution of the harmonic oscillator, which will lead us to define $u$ using the phase lag analysis of Section 3 .

\section{Harmonic oscillator}

As a first application towards testing the above techniques, the case of a simple harmonic oscillator is considered. This procedure is similar to the one proposed by [20], but it differs in the choice of the initial value problem used to calculate the phase lag of the present method. Towards this purpose, we consider the Lagrangian

$$
L(q, \dot{q})=\frac{1}{2} \dot{q}^{2}-\frac{1}{2} \omega^{2} q^{2}
$$




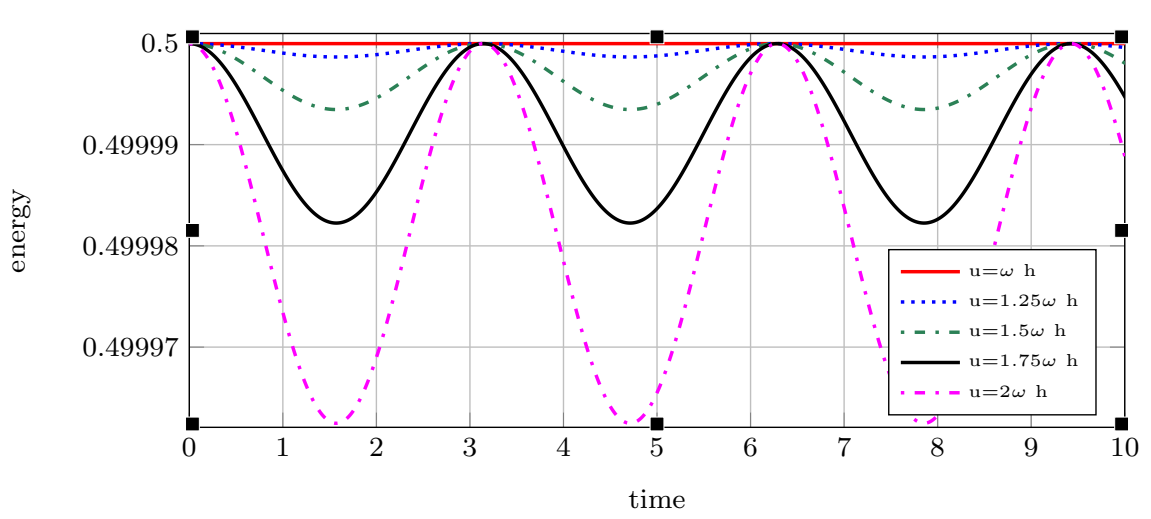

Fig. 3: Harmonic oscillator with frequency $\omega=1$, time step $h=0.01$ and $S=5$. Evolution of the total energy for different parameters $u$ in (22).

and the Euler-Lagrange equations

$$
\ddot{q}=-\omega^{2} q .
$$

Using the interpolation of (12), the discrete Lagrangian for the harmonic oscillator can be cast in the form

$$
\begin{aligned}
L_{d}\left(q_{k}, q_{k+1}\right)=\frac{h}{2}\left[\sum_{j=0}^{S-1} w^{j}\left(\dot{g}_{1}\left(t_{k}^{j}\right) q_{k}+\dot{g}_{2}\left(t_{k}^{j}\right) q_{k+1}\right)^{2}-\right. \\
\left.\omega^{2} \sum_{j=0}^{S-1} w^{j}\left(g_{1}\left(t_{k}^{j}\right) q_{k}+g_{2}\left(t_{k}^{j}\right) q_{k+1}\right)^{2}\right] .
\end{aligned}
$$

For this Lagrangian, the discrete Euler-Lagrange equations (3) give the twostep variational integrator

$$
q_{k+1}+\frac{\sum_{j=0}^{S-1} w^{j}\left[\dot{g}_{1}\left(t_{k}^{j}\right)^{2}+\dot{g}_{2}\left(t_{k}^{j}\right)^{2}-\omega^{2}\left(g_{1}\left(t_{k}^{j}\right)^{2}+g_{2}\left(t_{k}^{j}\right)^{2}\right)\right]}{\sum_{j=0}^{S-1} w^{j}\left[\dot{g}_{1}\left(t_{k}^{j}\right) \dot{g}_{2}\left(t_{k}^{j}\right)-\omega^{2} g_{1}\left(t_{k}^{j}\right) g_{2}\left(t_{k}^{j}\right)\right]} q_{k}+q_{k-1}=0 .
$$

Although the resulting integrator for the harmonic oscillator is explicit for any choice of the employed interpolation, in general, (27) can lead to implicit methods depending on the degree of nonlinearity in the problem and quadrature chosen in the discrete Lagrangian (for a discussion of implicit and explicit methods in the context of highly oscillatory problems, see [18]).

We now use the above integrator for different choices of functions $g_{1}$ and $g_{2}$ by addopting linear and cubic spline interpolations, described by (18) and (21), respectively. For the trigonometric interpolation of (22), we first test the 


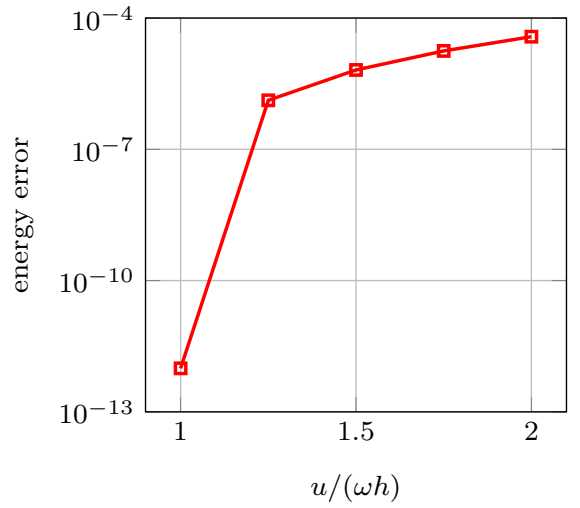

(a)

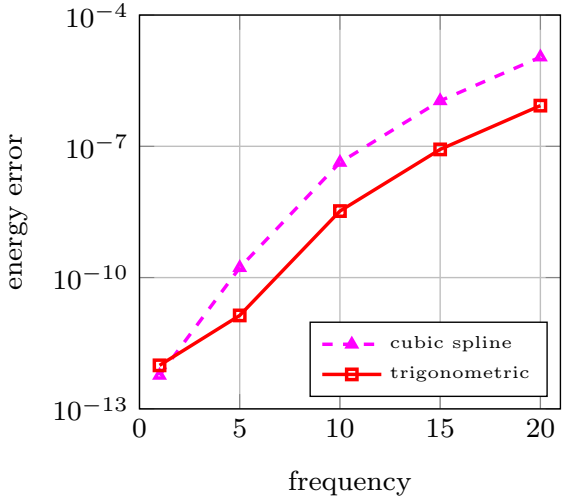

(b)

Fig. 4: Harmonic oscillator. (a): Energy error for trigonometric interpolation using for different choices of $u$ with $\omega=1, h=0.01$. (b): Energy error using cubic spline interpolation and trigonometric interpolation with $u=\omega h$ for the frequencies $\omega=1, \omega=5, \omega=10, \omega=15$ and $\omega=20$.

adoption of arbitrary values for the parameter $u$. Different results for these choices of $u$ will lead us to define this parameter analytically, see Section 6 .

Before testing the proposed high order integrator relying on trigonometric interpolation, we first integrate the harmonic oscillator by using the approaches presented in Section 4.1.

For $S=5$, i.e. 3 intermediate points in each time interval $\left[t_{k}, t_{k+1}\right]$, the numerical solution of the h.o. with $\omega=1$ and $h=0.01$ assuming initial conditions $\left(q_{0}, p_{0}\right)=(2,1)$ and using the linear interpolation (18) is investigated. The evolution of the total energy is presented in Figure 2(a) and compared with that obtained by using expressions (22). Even though both interpolations rely on the integrator (27), the resulting energy behavior by using trigonometric interpolation is much better. Furthermore, in Figure 2(b) the position error (i.e. the difference between the calculated and the exact solutions) is plotted for the trigonometric interpolation with $u=\omega h$ (red) and the linear interpolation (blue) illustrating that linear interpolation yields larger errors when compared to trigonometric interpolation. All the above results, when choosing $u=\omega h$, can be straightforwardly obtained and verified analytically as well (see Section 6).

The oscillations of the total energy for this kind of methods and for various choices of $u$, are illustrated in Figure 3. It is clear that for $u=\omega h$ the energy behavior is much better (this will be further discussed in Section 6).

For a more complete study of the important role which plays the parameter $u$ in the proposed numerical technique, we compute and plot the total energy error. For $u=\omega h$, in Figure 4(a) one can see that the energy error is close to $10^{-12}$, while the energy error of the trigonometric interpolation for different frequencies is depicted in Figure 4(b) together with the energy error of cubic 


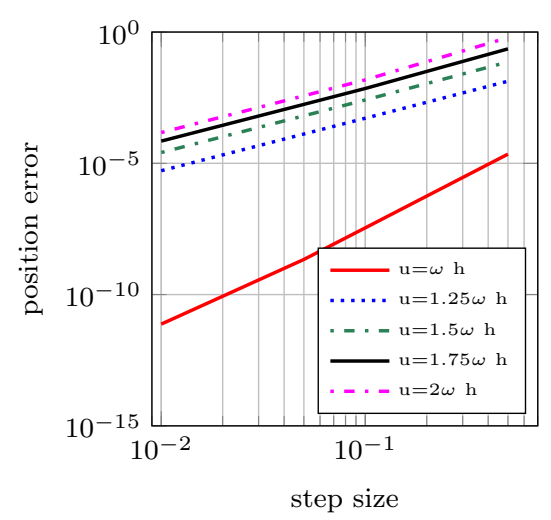

(a)

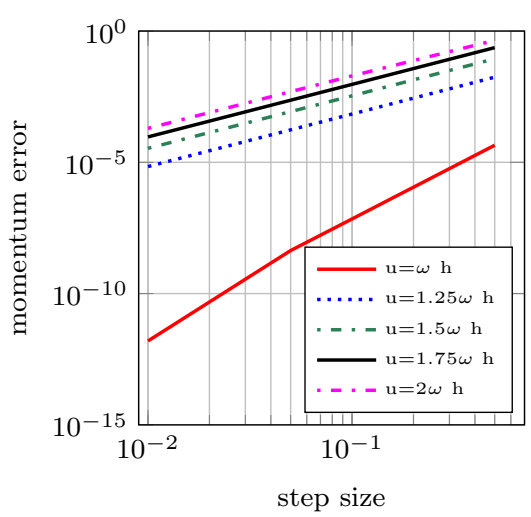

(b)

Fig. 5: Harmonic oscillator with $\omega=1$ and $S=5$. Global errors for (a): the position and (b): the momentum using four step sizes $h$ for different parameters $u$ in $(22)$.

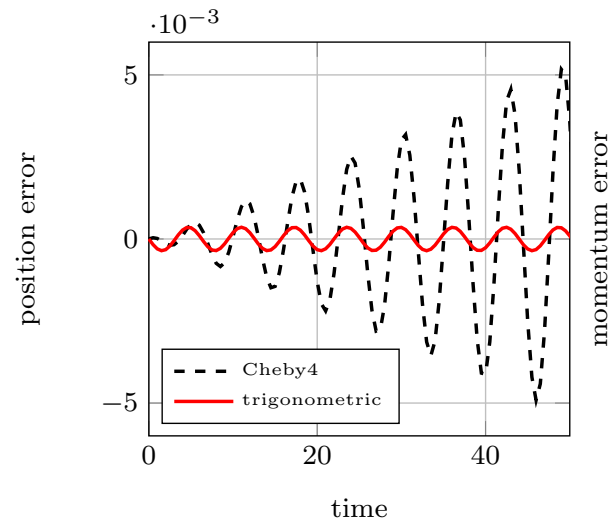

(a)

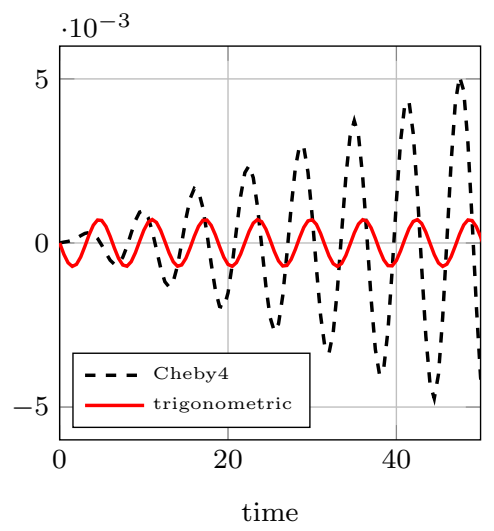

(b)

Fig. 6: Harmonic oscillator with $\omega=1$, using $h=0.5$ and $S=5$. (a): Evolution of error in the position for the Cheby4 of [19] and the trigonometric interpolation method with $u=\omega h$. (b): Evolution of error in the momentum for the Cheby4 of [19] and the trigonometric interpolation method for $u=\omega h$.

splines. Except for $\omega=1$, the phase fitted trigonometric interpolation behaves better than the cubic spline interpolation.

In order to illustrate the benefits of the phase-fitting technique on the numerical accuracy of the obtained methods we test the global errors for the position and momentum components at $t=3$, see Figure 5 . We choose time steps $h \in\{0.01,0.05,0.1,0.5\}$ and initial conditions $\left(q_{0}, p_{0}\right)=(2,1)$ and the different choices of $u$, as shown in Figure 3. One can imply that, as $u$ approaches 


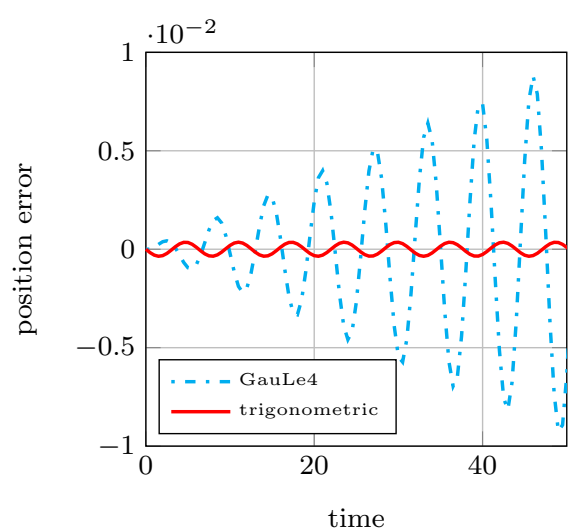

(a)

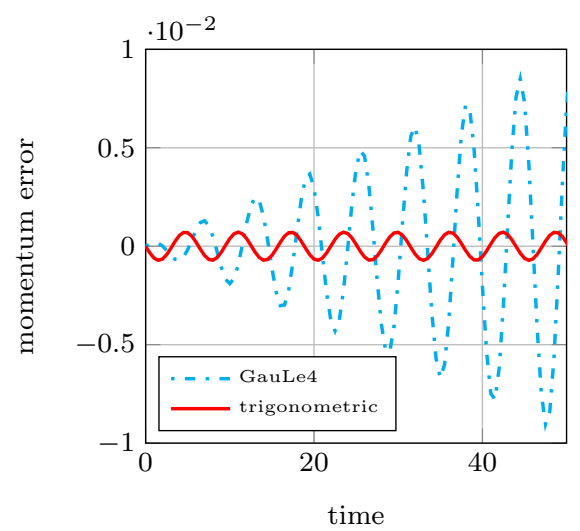

$(b)$

Fig. 7: Harmonic oscillator with $\omega=1$, using $h=0.5$ and $S=5$. (a): Errors in the position for the GauLe4 of [19] and the trigonometric interpolation method for $u=\omega h$. (b): Errors in the momentum for the Cheby4 of [19] and the trigonometric interpolation method for $u=\omega h$.

$\omega h$ from above, the accuracy of the method is increased. Furthermore, while the phase fitted method (red line) shows the order of accuracy 4 , choosing $u$ in a different (not phase-fitted) way (other lines) the order of accuracy decreases reaching, for this example, approximately the order 2, even though the same number of intermediate points is used.

For the sake of comparison, we further consider the fourth-order accuracy methods Cheby4 and GauLe4 derived by [19] who employed Chebyshev and Gauss-Legendre quadratures. In Figures 6 and 7, the evolution of error in the position $q$ and momentum $p$ are shown for the initial conditions $\left(q_{0}, p_{0}\right)=(2,1)$ and step size $h=0.5$. Obviously, the errors when using the trigonometric interpolation method (with $S=5$, fourth-order accuracy) are smaller (also bounded for all the integration time) than those obtained using Cheby 4 and GauLe4.

Finally, to explore the numerical convergence of the proposed method, we choose as initial conditions $\left(q_{0}, p_{0}\right)=(2,2)$ and the time interval $[0,3]$ as in [19]. The global errors for the position and momentum components at $t=3$ for time steps $h \in\{0.01,0.05,0.1,0.5,1\}$ are compared to those of [19], i.e. Cheby 4 and GauLe4 for the case of the harmonic oscillator with $\omega=1$, see Figure 8 . We remind that, all these methods are of order four which means that, for the same computational costs, the smallest errors in position and momentum are obtained with the method derived by using trigonometric interpolation for $u=\omega h$, see also Figure 9. We should stress, however, that all the results presented are independent of the particular initial conditions chosen. 


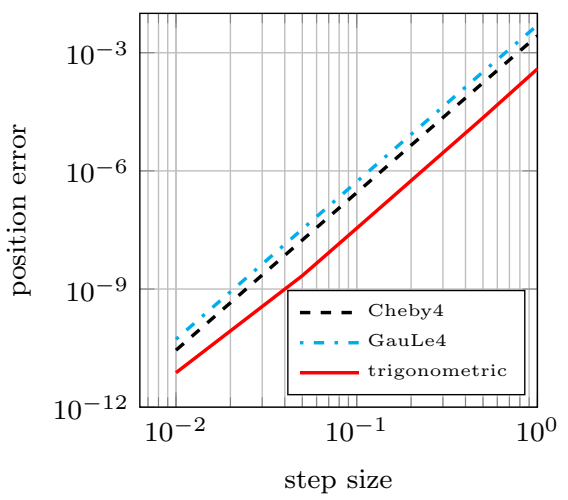

(a)

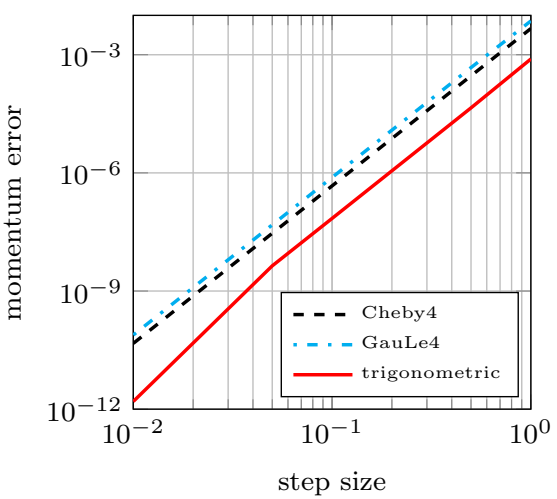

(b)

Fig. 8: Harmonic oscillator with $\omega=1$ and $S=5$. Global errors for (a): the position and (b): the momentum using five step sizes $h$ for the GauLe4, Cheby 4 of [19] and the trigonometric interpolation method.

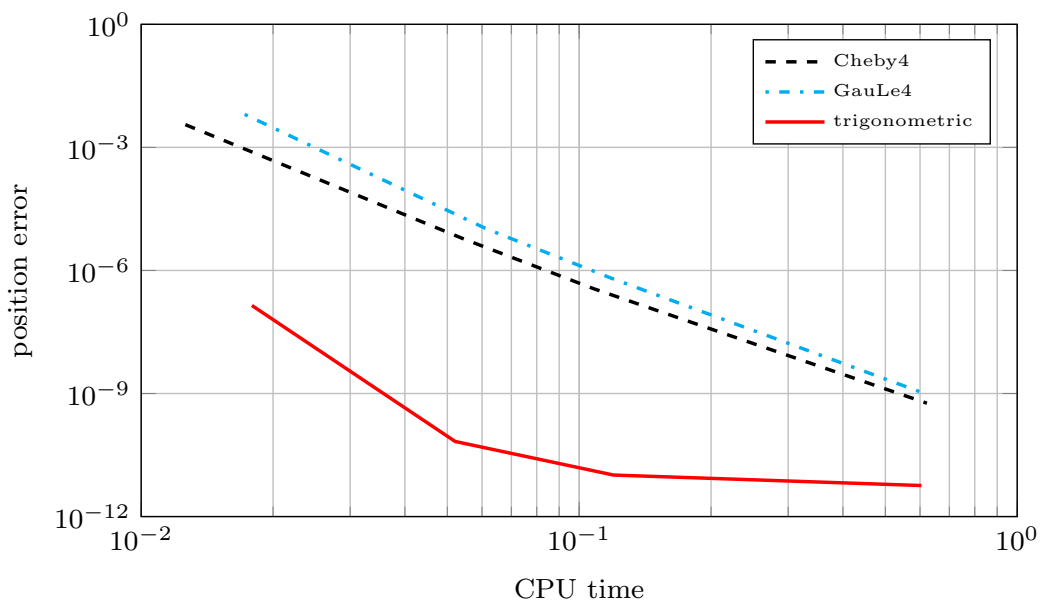

Fig. 9: Harmonic oscillator with $\omega=1$. Position error (absolute value) versus CPU time for methods using trigonometric interpolation for $S=5$ and for the GauLe4, Cheby4 of [19].

\section{Phase lag analysis}

In order to verify the optimal behavior of the method for the special choice of $u=\omega h$ (shown through the numerical results in the previous sections), in this section we use the definitions of the phase lag given in Section 3 to calculate the ratio of the estimated solution divided by the exact one at specific time instances. Then, following the works of $[10,16]$, a special initial value problem 
is considered, i.e. the harmonic oscillator with frequency $\omega$ and given positions $q(0)=\bar{q}_{0}, q(h)=\bar{q}_{1}$.

The ratio of the estimated solution $\hat{q}_{2}$ at time $2 h$, obtained via $(27)$, and the exact one as shown in (11), reads

$$
\frac{\hat{q}_{2}}{q(2 h)}=\frac{\Lambda(u, \omega, h) \bar{q}_{1}+\bar{q}_{0}}{\bar{q}_{0}-2 \cos (\omega h) \bar{q}_{1}}
$$

where

$$
\Lambda(u, \omega, h)=\frac{\sum_{j=0}^{S-1} w^{j}\left[\dot{g}_{1}\left(t_{k}^{j}\right)^{2}+\dot{g}_{2}\left(t_{k}^{j}\right)^{2}-\omega^{2}\left(g_{1}\left(t_{k}^{j}\right)^{2}+g_{2}\left(t_{k}^{j}\right)^{2}\right)\right]}{\sum_{j=0}^{S-1} w^{j}\left[\dot{g}_{1}\left(t_{k}^{j}\right) \dot{g}_{2}\left(t_{k}^{j}\right)-\omega^{2} g_{1}\left(t_{k}^{j}\right) g_{2}\left(t_{k}^{j}\right)\right]} .
$$

From the latter expressions, it becomes clear that for exponentially fitted methods, the condition

$$
\Lambda(u, \omega, h)=-2 \cos (\omega h) .
$$

must hold. For the special case of the exact solution $e^{i \omega t}$, the initial conditions are

$$
q(0)=\bar{q}_{0}=1, \quad q(h)=\bar{q}_{1}=e^{i \omega h} .
$$

Thus, inserting (29) and (31) into (28), the phase lag of the method, obtained according to (11), takes the form

$$
\ell(\omega h)=\arctan \left(\frac{\Lambda(u, \omega, h) \sin (\omega h)+\sin (2 \omega h)}{-\Lambda(u, \omega, h) \cos (\omega h)-\cos (2 \omega h)}\right) .
$$

By demanding the phase lag in the latter expression to be zero, using the relation $\sin (2 \omega h)=2 \sin (\omega h) \cos (\omega h)$ we conclude that either (30) must hold or $\sin (\omega h)=0$. This means that, keeping the phase lag zero when using (30), the resulting amplification error is $\alpha=1$ and, hence, exponentially fitted methods are created, as shown e.g. in [14]. In addition, from (29) and (30) the determined parameter $u$ is $u=\omega h$.

\begin{tabular}{|c|c|c|c|c|}
\hline \multicolumn{5}{|c|}{ phase lag $\ell(\omega h)$} \\
\hline frequency & $S$ & linear & cubic & trigonometric $u=\omega h$ \\
\hline$\omega=1$ & 3 & $-8.33294 e-12$ & $-2.42861 e-17$ & 0 \\
$\omega=10$ & 5 & $-8.29447 e-07$ & $-2.77840 e-10$ & 0 \\
$\omega=50$ & 8 & $-2.30817 e-03$ & $-1.45884 e-06$ & 0 \\
\hline
\end{tabular}

Table 1: Harmonic oscillator with $h=0.01$ : the calculated phase lag for integrators using interpolation techniques for three different frequencies.

In Table 1, the calculated phase lag for integrators using linear, cubic and trigonometric interpolation with $u=\omega h$ is shown for the numerical solution of 
the harmonic oscillator in three different frequencies $\omega$ and $h=0.01$. Moreover, we have checked different values of intermediate points $S$ for cases of high frequencies in order to keep the resulting $\ell(\omega h)$ rather small when using linear and cubic interpolation.

6.1 Frequency estimation for mass points motion in three dimensions

In our previous work [16], we constructed adaptive time step variational integrators using phase fitting techniques and estimated the required frequency through the use of a harmonic oscillator with given frequency $\omega$. Here, in solving the general $N$-body problem by using a constant time step, a new frequency estimation is necessary in order to find for each body i) the frequency at an initial time $t_{0}$ and ii) the frequency at time $t_{k}$ for $k=1, \ldots, N-1$.

It is now clear that, by applying the trigonometric interpolation (23), the parameter $u$ can be chosen as $u=\omega h$. For problems for which the domain of frequency $\omega$ is fixed and known (such as the harmonic oscillator) the parameter $u$ can be easily computed. For the solution of orbital problems involved in the general $N$-body problem, where no unique frequency is determined, the parameter $u$ must be defined by estimating the frequency of the motion of any moving material point.

Towards this purpose, we consider the general case of $N$ masses moving in three dimensions. If $q_{i}(t)(i=1, \ldots, N)$ denotes the trajectory of the $i$-th particle, its curvature can be computed from the known expression

$$
k_{i}(t)=\frac{\left|\dot{q}_{i}(t) \times \ddot{q}_{i}(t)\right|}{\left|\dot{q}_{i}(t)\right|^{3}},
$$

where $\dot{q}_{i}(t)$ the velocity of the $i$-th mass with magnitude $\left|\dot{q}_{i}(t)\right|$ at a point $q_{i}(t)$. After a short time $h$, the angular displacement of that mass is $h \mid \dot{q}_{i}(t) \times$ $\left.\ddot{q}_{i}(t)|/| \dot{q}_{i}(t)\right|^{2}$, which for each mass's actual frequency gives the expression

$$
\omega_{i}(t)=\frac{\left|\dot{q}_{i}(t) \times \ddot{q}_{i}(t)\right|}{\left|\dot{q}_{i}(t)\right|^{2}} .
$$

From $(33)$ and (34) the well known relation $\omega_{i}(t)=k_{i}(t)\left|\dot{q}_{i}(t)\right|$ holds (see also $[16])$.

For the specific case of many-particle physical problems, that can be described via a Lagrangian of the form $L(q, \dot{q})=\frac{1}{2} \dot{q}^{T} M(q) \dot{q}-V(q)$, where $M(q)$ represents a symmetric positive definite mass matrix and $V$ is a potential function, the continuous Euler-Lagrange equations are $M(q) \ddot{q}=-\nabla V(q)$. In this case, the expression for frequency estimation (34), referred to the $i$-th body at time $t_{k}, k=1, \ldots, N-1$, takes the form

$$
\omega_{i}\left(t_{k}\right)=h^{-1} \frac{\left|M^{-1}\left(q_{k}\right) p_{k} \times\left(M^{-1}\left(q_{k}\right) p_{k}-M^{-1}\left(q_{k-1}\right) p_{k-1}\right)\right|}{\left|M^{-1}\left(q_{k}\right) p_{k}\right|^{2}},
$$

where the quantities on the right hand side are the mass matrix, the configuration and the momentum of the $i$-th body. Since the frequency $\omega_{i}\left(t_{k}\right)$ must 
be also known at an initial time instant $t_{0}$ (in which the initial positions are $\bar{q}_{0}$ and initial momenta are $\bar{p}_{0}$ ), using the continuous Euler-Lagrange equation at $t_{0}$ we obtain

$$
\omega_{i}\left(t_{0}\right)=\frac{\left|M^{-1}\left(\bar{q}_{0}\right) \bar{p}_{0} \times\left(-M^{-1}\left(\bar{q}_{0}\right) \nabla V\left(\bar{q}_{0}\right)\right)\right|}{\left|M^{-1}\left(\bar{q}_{0}\right) \bar{p}_{0}\right|^{2}} .
$$

Equations (35) and (36) provide an "estimated frequency" for each mass in the general motion of the $N$-body problem. This allows us to derive high order variational integrator methods using trigonometric interpolation where the frequency is estimated at every time step of the integration procedure. These methods show better energy behavior, i.e. smaller total energy oscillation than other methods which employ constant frequency, see Sections 7 and 8 .

Before closing this section, it should be mentioned that the linear stability of our method is comprehensively analyzed in our previous works [25,24].

\section{Orbital problems - N-body problem}

In this section, we focus on the numerical solution of the general $N$-body problem. To this end, the Lagrangian function according to $[5,6]$ is written as

$$
L(q, \dot{q})=\frac{1}{2} \sum_{i=1}^{N} m_{i} \dot{q}_{i}^{2}+\sum_{i=1, j=1, i \neq j}^{N} G \frac{m_{i} m_{j}}{\left\|q_{i}-q_{j}\right\|}
$$

where $G$ denotes the gravitational constant. In the following subsections we describe the motion of planar two body problem and that of the multi-body solar system in conjunction with novelties of the present work.

\subsection{Planar two body problem}

We first test the above techniques in the study of the simple problem of two objects mutually interacting through a central force. The most famous example of this type is the planar Kepler problem (also called the two body problem) that describes the motion of two masses attracting each other with the gravitational force. In the solar system, the gravitational interaction between two bodies leads to elliptic orbits for planets and hyperbolic orbits for comets.

By choosing one of the bodies (the heavier) as the center of the coordinate system, the motion remains planar. Denoting the position of the second body by $q=\left(q_{1}, q_{2}\right) \in \mathbb{R}^{2}$, the Lagrangian (37) for the system, assuming the masses of the bodies and the gravitational constant are equal to 1 , takes the simple form

$$
L(q, \dot{q})=\frac{1}{2} \dot{q}^{2}+\frac{1}{|q|}
$$




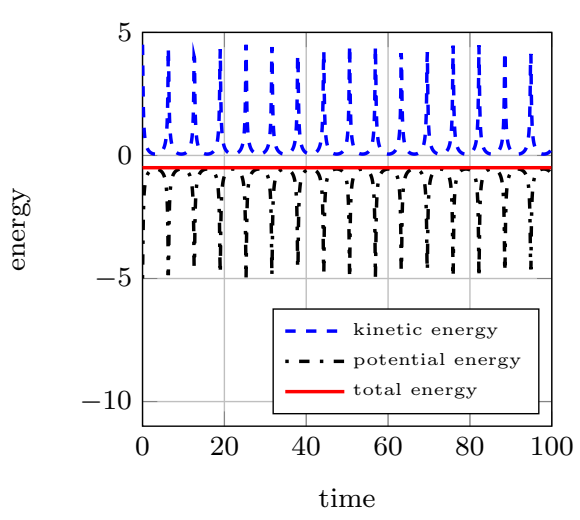

(a)

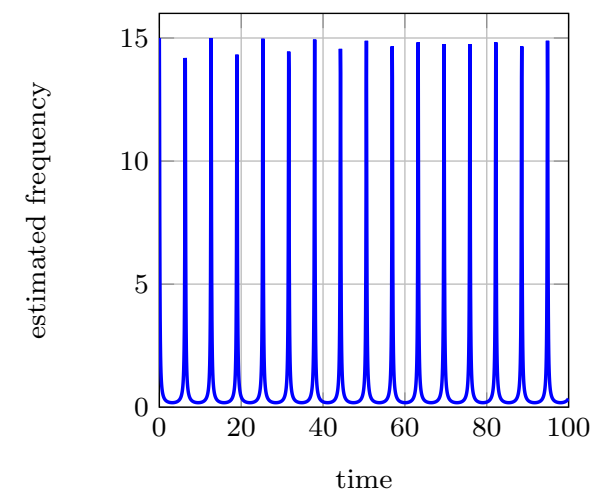

$(b)$

Fig. 10: Planar two body problem with $\epsilon=0.8, h=0.01$ and $S=5$ for $10^{4}$ steps. (a): Energy evolution using trigonometric interpolation. (b): The estimated frequency for the moving mass using the expression (34).

As initial conditions we assume

$$
q=(1-\epsilon, 0), \dot{q}=\left(0, \sqrt{\frac{1+\epsilon}{1-\epsilon}}\right)
$$

where $\epsilon \in \mathbb{R}$ is the eccentricity of the orbit, see [5].

In the first computational experiment, we consider the eccentricity $\epsilon=$ 0.8 and the time step $h=0.01$. The good energy behavior of the method using trigonometric interpolation with $u=\omega h$ and $S=5$ is concluded from Figure 10(a) obtained for a simulation of $10^{4}$ steps. As mentioned before, the frequency is estimated at every integration step using (34)-(35) and its time variation is shown in Figure 10(b). The peaks represent the estimated frequency at points close to perihelion, i.e. the point where the moving mass is nearest to the central body. Furthermore, a comparison of the total energy evolution using constant (dashed line) and an estimated (solid line) frequency is shown in Figure 11. As can be seen, even for relatively small eccentricities (case of $\epsilon=0.2$ ) the amplitude of the energy oscillation is smaller when the frequency is estimated at every time step of the integration process. Similar results can be obtained for higher eccentricities.

Finally, in the last experiment, we integrate the two-body problem with eccentricities $\epsilon=0.6, \epsilon=0.7$ and $\epsilon=0.8$ for $10^{3}$ time steps in order to check the need of a high order variational scheme. For that we test the long term behavior of two methods, the trigonometric and the Störmer-Verlet method, see [5], which is also variational, but of second order accuracy. For both methods, the results for the configuration $q_{k}$ of the body's orbit are illustrated in Figure 12. In both cases, the results demonstrate the excellent behavior of the higher accurate proposed method, even for orbits with extremely high eccentricity 


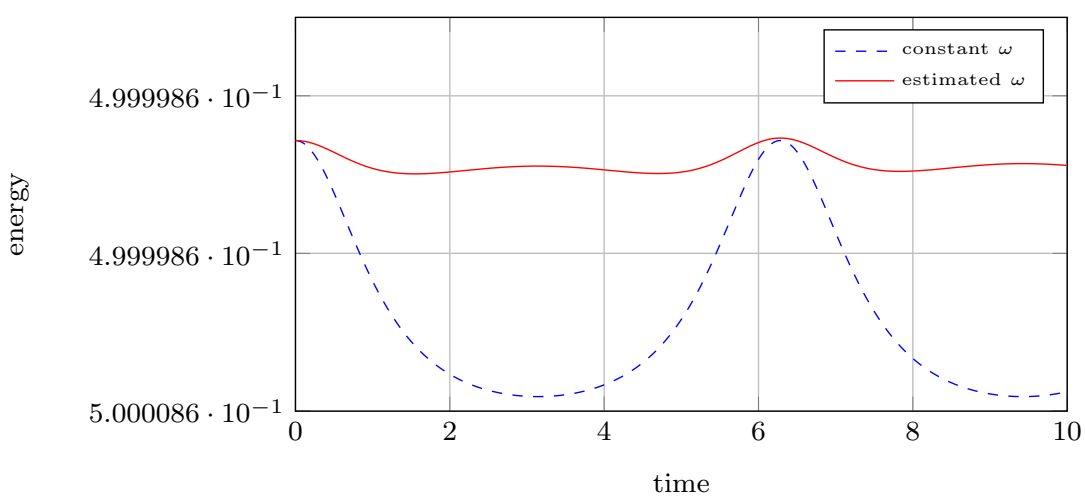

Fig. 11: Planar two body problem with $\epsilon=0.2$ using trigonometric interpolation for $S=5, h=0.01$. Evolution of the total energy for constant and estimated frequency using (34).

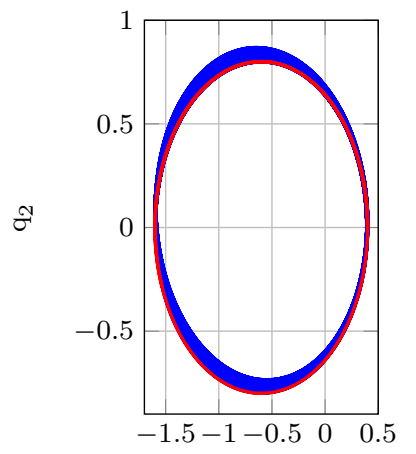

$\mathrm{q}_{1}$

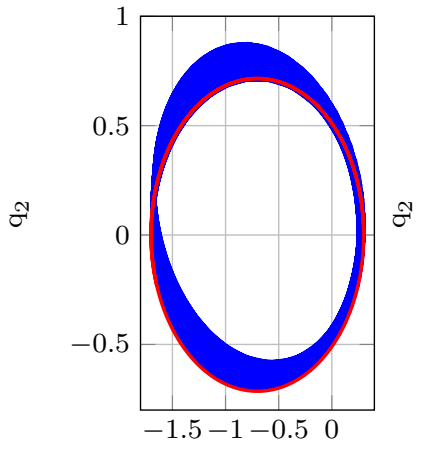

$\mathrm{q}_{1}$

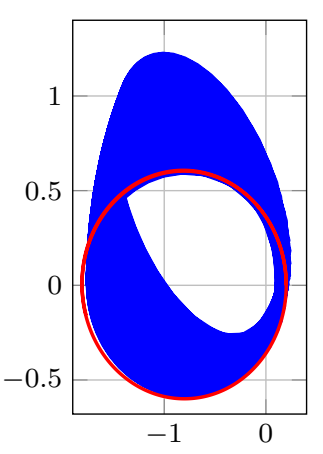

$\mathrm{q}_{1}$

(a)

(b)

Fig. 12: Planar two body problem for $h=0.01$ for $10^{3}$ steps for eccentricities (a): $\epsilon=0.6,(\mathrm{~b}): \epsilon=0.7$ and (c): $\epsilon=0.8$. Long term behavior of the StörmerVerlet method of [5] and the one that uses trigonometric interpolation with $S=5$.

and large number of periods. On the other hand, the solution using StörmerVerlet, creates perturbed orbits, even for small eccentricities, stating the need of high order schemes.

\subsection{Modified solar system}

In order to illustrate the advantage of choosing the parameter $u$ via an estimated frequency (34) for multi-body systems, we adopt the modified solar 


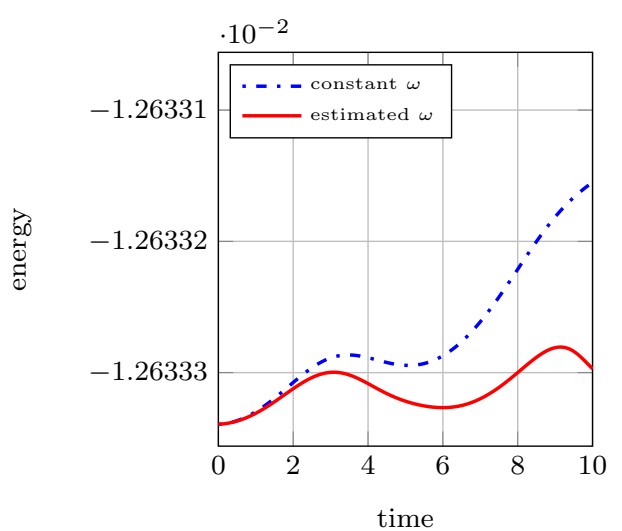

$(a)$

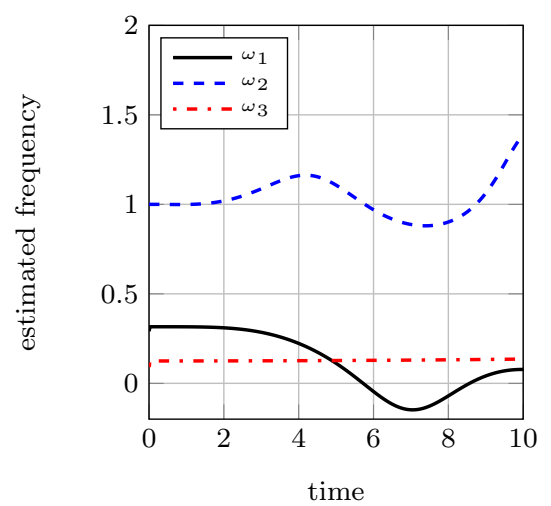

$(b)$

Fig. 13: Modified solar system with $h=0.01$ and $S=3$. (a): Total energy evolution using trigonometric interpolation for constant $\omega_{i}$ (blue line) and estimated $\omega_{i}$ at every time step (black line). (b): Estimated frequency for the three bodies of the solar system problem using the expression (34).

system with two planets, see [5]. For this system, which is described by the Lagrangian function (37) for $N=3$, we assume masses $m_{1}=1, m_{2}=m_{3}=10^{-2}$, initial configurations $q_{1}=(0,0), q_{2}=(1,0), q_{3}=(4,0)$ and initial velocities $\dot{q}_{1}=(0,0), \dot{q}_{2}=(0,1), \dot{q}_{3}=(0,0.5)$. The obtained motion of the two planets is nearly circular with periods equal to about $2 \pi$ and $14 \pi$ respectively, see [5].

At first we test the variational integrator using trigonometric interpolation with constants $\omega_{i}, i=1,2,3$, during the integration procedure, versus the one derived by estimating the parameter $u$ at every time step with (34). In Figure 13(a), we compare the variation of the total energy resulting in these two methods. The advantage of estimating the frequency at every step is obvious. In Figure 13(b) the evolution of the estimated angular velocities for each body $\left(\omega_{1}, \omega_{2}\right.$ and $\omega_{3}$ respectively) is shown. For both numerical tests, the number of intermediate points is $S=3$ while the time step is $h=T_{1} / 365$, with $T_{1}=2 \pi$ being the period of the first planet.

\section{Solar system}

As a last example, we examine the applicability of the proposed variational integration schemes on the numerical solution of the complete solar system, see [5]. The problem consists of a nine planet system, Mercury (Me), Venus (V), Earth (E), Mars (Ma), Jupiter (J), Saturn (Sa), Uranus (U), Neptune (N) and Pluto $(\mathrm{P})$ all orbiting around the Sun $(\mathrm{Su})$ plus the Moon (Mo) (here we also consider Moon's orbit around Earth). For this general $N$-body (with $N=11$ ) problem, the initial conditions (positions and velocities) may be taken from 


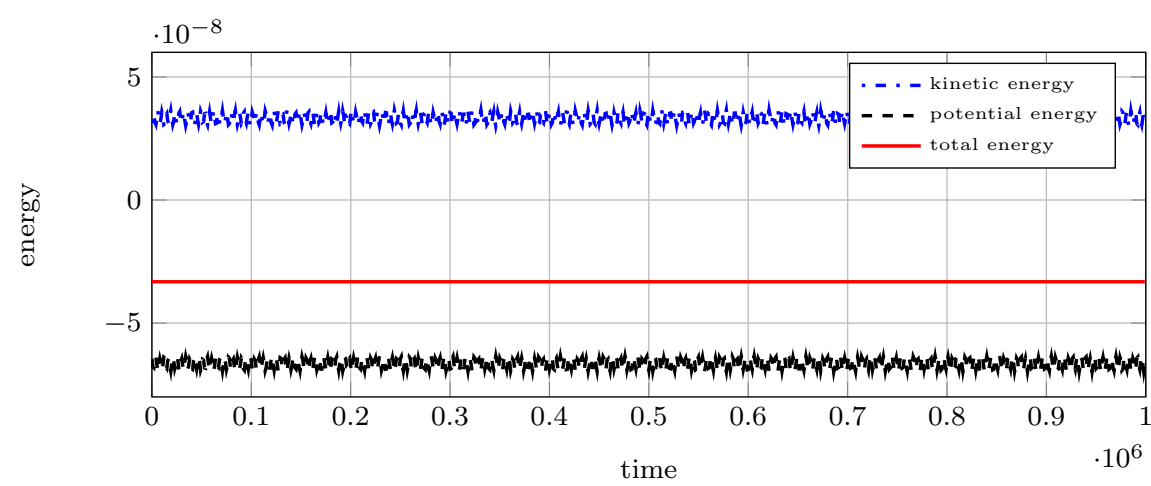

Fig. 14: Kinetic, potential and total energy evolution for the numerical integration of the solar system problem for 1 million days, using trigonometric interpolation with $S=3$ and $h=1$ day.

Nasa's JPL data base, while the Lagrangian of the system is given by (37) for $N=11$ and $G=2.95912208286 \cdot 10^{-4}$.

By using trigonometric interpolation functions with time step equal to one (Earth) day for $S=3$ intermediate points, the evolution of the kinetic, potential and total energy of the system for $10^{6}$ days (the time step is rather big compared to the period of motion of the planets orbiting close to the Sun) is demonstrated in Figure 14, from where one may conclude that, even for long term integration processes, the energy behavior is good and the level of stability is high.

It is worth mentioning that, for all the above results, the parameters $\omega_{i}, i=$ $1, \ldots, 11$ are estimated at every time step as it is plotted in Figure 15. As can be seen, the resulting frequency of the Suns motion is not so stable (this is due to the type of the Sun's motion). For all other planets, the estimated frequency is remarkably stable, even for a great number $\left(10^{6}\right)$ of step integration days.

\section{Summary and conclusions}

In the present paper, a general method for the derivation of high order variational integrators is presented. The resulting integrators inherit geometric properties of variational integrators and by using linear and cubic interpolations allow us to derive advantageous high order methods. Furthermore, as a special case, integrators relying on trigonometric interpolation are obtained, suitable for problems with oscillating behavior. These methods depend on a parameter $u$ which can be determined on the basis of phase lag analysis theory.

The proposed method has better energy behavior, i.e. the bounds on the total energy oscillations are much smaller compared to the standard ones and to the similar but not phase fitted ones of [19]. In addition, our phase fitted variational integrators show better accuracy compared to the non phase fitted 


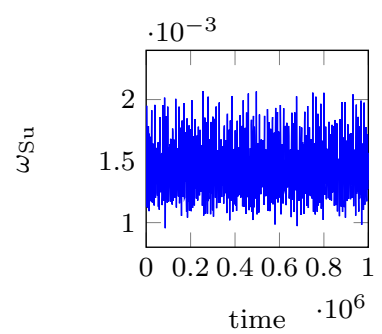

(a) Sun

$\frac{\text { 뙤 }}{3}$

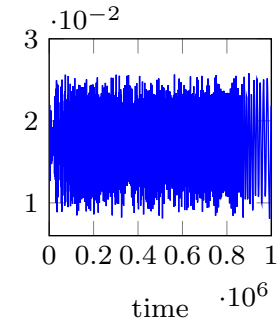

(d) Earth

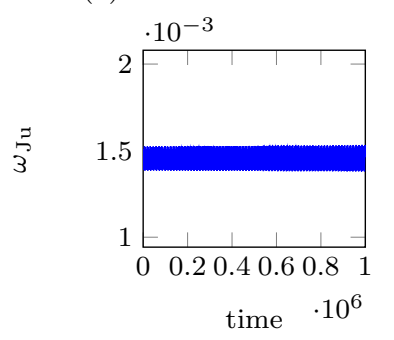

(g) Jupiter

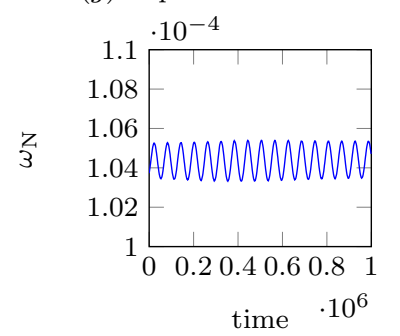

(j) Neptune

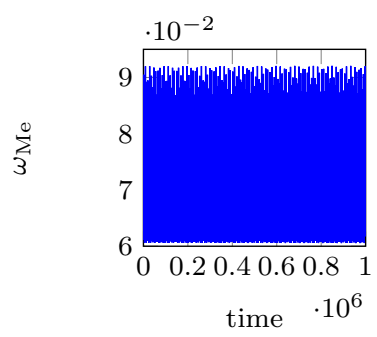

(b) Mercury

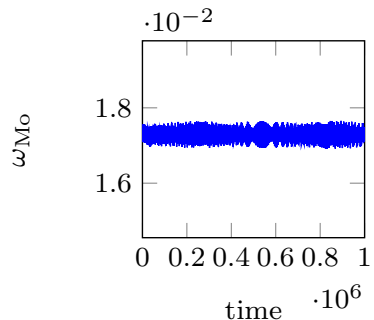

(e) Moon

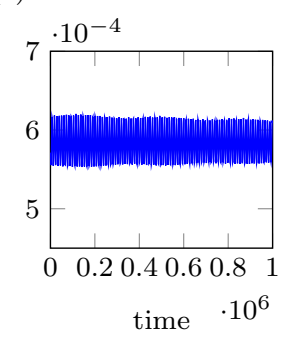

(h) Saturn

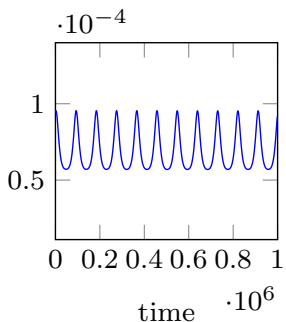

(k) Pluto

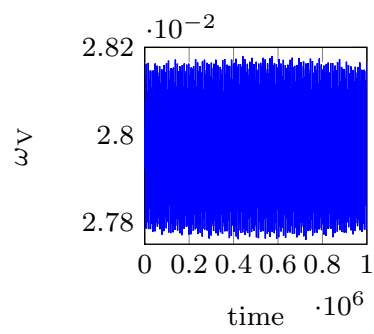

(c) Venus

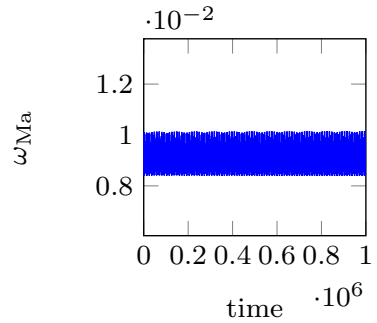

(f) Mars

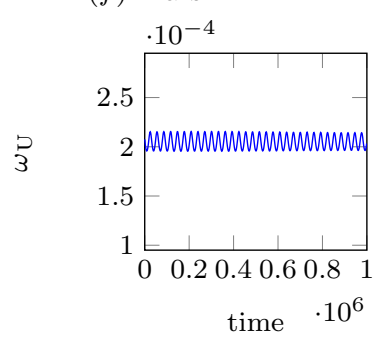

(i) Uranus

Fig. 15: The estimated frequency (34) for the numerical integration of the complete solar system problem for $10^{6}$ days, using trigonometric interpolation with $S=3$ and $h=1$ day for all the planets. 
ones of the same order. Moreover, the frequency $\omega$ is estimated using an expression of the angular velocity of a body's motion offering a great advantage for numerical solutions of many-body problems.

\section{References}

1. Engquist, B. \& Fokas, A. \& Hairer, E. \& Iserles, A. (2009), Highly oscillatory problems. Cambridge: Cambridge University Press.

2. Wendlandt, J. \& Marsden, J. (1997), Mechanical integrators derived from a discrete variational principle. Physica D, 106, 223-246.

3. Kane, C. \& Marsden, J. \& Ortiz, M. (2001), Symplectic-energy-momentum preserving variational integrators. Journal of Mathematical Physics, 40, 3353-3371.

4. Marsden, J. \& West, M. (2001), Discrete mechanics and variational integrators. Acta Numerica, 10, 357-514.

5. Hairer, E. \& Lubich, C. \& Wanner, G. (2003), Geometric numerical integration illustrated by the Störmer-Verlet method. Acta Numerica, 12, 399-450.

6. Leimkuhler, B. \& Reich, S. (2004), Simulating Hamiltonian dynamics. Cambridge: Cambridge Monographs on Applied and Computational Mathematics.

7. Ober-Blöbaum, S. \& Saake, N. (2015), Construction and analysis of higher order Galerkin variational integrators. Advances in Computational Mathematics, 41, 955986.

8. Campos, C.M. \& Ober-Blöbaum, S. \& Trélat, E. (2015), High order variational integrators in the optimal control of mechanical systems. Discrete and Continuous Dynamical Systems A, 35, 4193-4223.

9. Ober-Blöbaum, S. (2017), Galerkin variational integrators and modified symplectic Runge-Kutta methods. IMA J. Numer. Analysis, 37, 375-406.

10. Brusca, L. \& Nigro, L. (1980), A one-step method for direct integration of structural dynamic equations. Internat. J. Numer. Methods Engnr., 15, 685-699.

11. Van de Vyver, H. (2006), A fourth-order symplectic exponentially fitted integrator. Computer Physics Comm., 174, 255-262.

12. Gautschi, W. (1961), Numerical integration of ordinary differential equations based on trigonometric polynomials. Numer. Math., 3, 381-397.

13. Lyche, T. (1972), Chebyshevian multistep methods for ordinary differential equations. Num. Math., 19, 65-75.

14. Ixaru, L. Gr. \& Vanden Berghe, G. (2004), Exponential Fitting.Dordrecht: Kluwer Academic Publishers.

15. Cappiello, M. \& Nicola, F., (2012), Regularity and decay of solutions of nonlinear harmonic oscillators. Advances in Mathematics 229, 1266-1299.

16. Kosmas, O.T. \& Vlachos. D.S. (2010), Phase-fitted discrete Lagrangian integrators. Computer Physics Comm., 181, 562-568.

17. Kosmas, O.T. \& Leyendecker, S. (2012), Phase lag analysis of variational integrators using interpolation techniques. PAMM Proc. Appl. Math. Mech., 12, 677-678.

18. Stern, A. \& Grinspun, E. (2009), Implicit-explicit integration of highly oscillatory problems. SIAM Multiscale Modeling and Simulation, 7, 1779-1794.

19. Leok, M. \& Zhang, J. (2011), Discrete Hamiltonian variational integrators. IMA Journal of Numerical Analysis, 31, 1497-1532.

20. Lambert, J.D. \& Watson, I.A. (1976), Symmetric multistep methods for periodic initial value problems. IMA Journal of Applied Mathematics, 18, 189-202.

21. Press, W.H. \& Teukolsky, S.A. \& Vetterling, W.T. \& Flannery, B.P. (1992), Numerical recipes in $C$ : the art of scientific computing. Cambridge: Cambridge University Press.

22. Kosmas, O.T. \& Vlachos, D.S. (2012), Local path fitting: a new approach to variational integrators. Journal of Computational and Applied Mathematics, 33, 2632-2642.

23. Kosmas, O.T. (2011), Charged particle in an electromagnetic field using variational integrators. ICNAAM Numerical Analysis and Applied Mathematics, 1389, 1927-1931.

24. Kosmas, O.T. \& Leyendecker, S. (2016), Analysis of higher order phase fitted variational integrators. Advances in Computational Mathematics, 42, 605-619. 
25. Kosmas, O.T. \& Leyendecker, S. (2014), Stability analysis of high order phase fitted variational integrators. Proceedings of WCCM XI - ECCM V - ECFD VI, 1389, 548556. 